\title{
REAL TIME TELETEXT BROADCAST SYSTEM PERFORMANCE ENHANCEMENT USING GHOST ROWS
}

\author{
Y.M. Siu, C.K. Chan and *K.L. Ho \\ Department of Electronic Engineering, \\ City University of Hong Kong, Tat Chee avenue, \\ Kowloon, Hong Kong. \\ *Department of Electrical and Electronic Engineering, \\ The University of Hong Kong, \\ Pokfulam Road, Hong Kong.
}

\begin{abstract}
$\underline{\text { Abstract }}$
Teletext is a one way broadcast system which provides a variety of services to its users. A one way broadcast system is attractive because a single transmission of a piece of information satisfies all potential users of that information. The main drawback of these types of systems is that a user must wait until the required information arrives. Therefore, the access time is an important consideration in the design of broadcast teletext systems. In addition, another important factor, information update delay, is very significant in real time broadcast teletext. The shortening of this delay is important for real time applications especially for systems broadcasting financial information. In this paper, we propose to use the ghost rows together with the storage capabilities of modern terminals to shorten both the access time and the update delay. A queueing model is developed to analyse the performance of this new system.
\end{abstract}

\section{Introduction}

Teletext is a one-way information delivery system which provides a variety of services to its users. These include information retrieval, software distribution, advertisement etc. Information is organized into units called pages. Pages of information are broadcast to all users in a continuous manner [1][2]. The configuration of a typical teletext system is shown in Fig. 1. The service computer is connected to the user terminals by a one-way communication network. The server computer maintains a database, and information pages in the database are updated regularly by service providers. When a page of information is requested by a user, the user terminal examines the broadcast data until the desired page is detected. This page is then captured, stored, and displayed. Since the request does not actually reach the service computer, such a feature may be described as pseudo interactive. Although teletext does not offer real interactive information services due to the oneway nature, it has the attractive feature of being able to support an unlimited number of users with no effect on system access time performance. With interactive systems, the system performance degrades quickly with load [1]. The quality of service provided to a teletext user depends on various aspects of the system. These include information content, ease of access, access time and update delay. The first two factors are being taken care of by the service providers and the decoder chip designers. In this paper, we concentrate on improving access time and update delay. Access time is defined as the elapse time between a user request for a page and the next transmission of that page. Update delay is defined as the delay time between updates or changes received in the information center and the updates received by the users. Access time to obtain the requested page is one of the major performance criteria for a teletext service. If a user has to wait for too long, he may become impatient and not want to use the system. The shortening of the update delay was not important previously because the teletext was only used to broadcast TV program time tables, advertisements, news etc. which are quite static. This is not the case for some modern systems broadcasting real time information such as financial data. Currently, Dow Jones of USA and Stock Exchange of Hong Kong are using teletext as one of the mediums to deliver information on stocks, bonds, options, current financial news, etc. which are very dynamic. Both the access time and the update delays are extremely important to these systems. This is because the remote users would like to have the most up-todate information as though as they were inside the trading center.

The fundamental nature of broadcast teletext transmissions imposes certain constraints on obtaining instant access and updates. Factors contributing to these include transmission speeds, efficiency of page encoding and decoding methods and broadcast schedules. Both the transmission speeds and coding methods were determined when the system and decoder chips were designed. In this paper, we propose to use the ghost rows (extension packets) together with the storage capabilities of modern terminals to imptove both the access time and the update performance. However, the new system is still compatible to users with older terminals. For details of the functions of the ghost rows, please refer to [3][4].

In section II, we point out the disadvantages of some existing methods using broadcast schedules to shorten the access time. In section III, we introduce our model using Ghost rows (extension packets) and local memory for data storage. Section IV contains the analysis of our model. Some numerical results are presented in Section $V$.

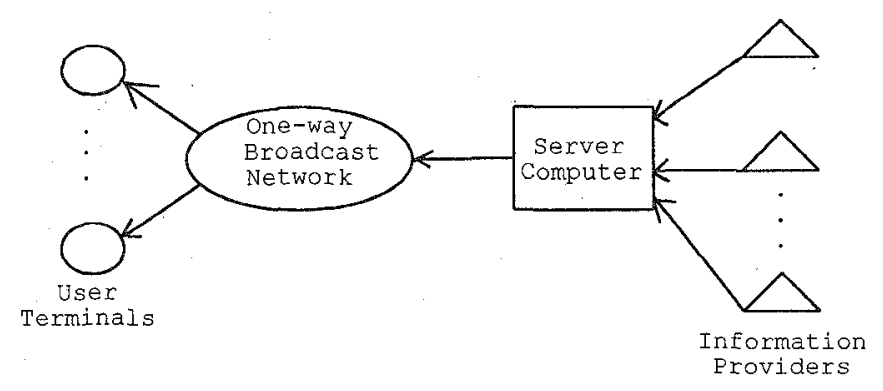

Fig. 1 A typical Teletext System 


\section{Existing Methods}

In [5], a probabilistic page broadcasting schedule was analysed. The update delay of this model is the same as the access delay because any changes in a page have to rely on the next transmission of that page. The main drawback of this random sequence broadcast schedule is that both the access time and update delay may become unbound. However, the system access time and updates at any time are expected to reach some limits for the system to be viable. Also, it may not be possible to determine exactly which pages are the more popular ones.

In [5][6], a cyclic page broadcasting method with improved performance was investigated. The minimization of the overall average access time is due to the effect of reducing the access time for the most popular pages at the expense of the access time for the less popular ones. That means, for users often needing to retrieve those 'non-popular' pages, this would be totally unacceptable. Also, for systems broadcasting financial information, the 'popular' pages may vary from time to time and may be affected by many unforeseeable factors. Therefore, this method is considered unsuitable.

Another method, the linked pages scheme, each page contains an ordered list of linked pages in extension packets 27 [3]. This list identifies the pages that are most likely to be requested next by the user. After a requested page is received, the user terminal prefectures those pages using the link list and stores them in the memory. If the request is from one of the stored pages, the response time will be almost instantaneous. This method significantly reduces the average access time if information pages in the database are related. But for systems where the selection of each page is independent from any other page, such as financial information, this method is not valid. Also, this method does not have any effect on improving the update delay.

\section{System Model Overview}

In the model we proposed, all the pages will have the same mean access time and update delay. This is important for real time information especially financial information if all the users are to be satisfied.

All user terminals are assumed to have a mass storage facility, this is made possible with the recent advances in computer technology that have led to the development of inexpensive modern PC/terminals [7]. This equipment provides users with inexpensive mass storage systems which were not possible a few years ago. The key to reducing the access time is to use more memory and capture pages in advance. If the memory is large enough and stores all the information pages from a data base cycle, the waiting time will be just the data retrieving time from the local memory, which is negligible. For example, 4000 teletext pages require less than $4 \mathrm{MB}$ memories. Even if the memory capacity is insufficient to store the entire database to guarantee instant access, it still features attractive access time reduction if only a part of the data base is kept.

The strategy to shorten the update delays is based on the fact that the data changing rate of teletext is much slower than its data transmission rate [8]. Each packet of all pages is considered as one unit. If changes occur on any of the packets as a result of information update, these packets would be sent using the ghost rows (extension packets) of each page. Row numbers $24,25,29$ or 31 are suitable for this purpose [3]. If the updates of a page reach the users before the next transmission of that page, the delay would be reduced.

\section{Performance Analysis}

\section{A. Reduction of Access Times}

Let the number of pages in the database be $\mathrm{N}$ and the original transmission rate be $\mu_{0}$ pages per second. Each page is transmitted once in each broadcasting cycle so that the access time is the same for every page. A simple example of a broadcast cycle is $(1,2,3 \ldots N)$. The maximum access time is the same as the cycle time.

$$
T_{\max }=T_{c y c}=N / \mu_{0}
$$

The average access time is

$$
T_{\text {average }}=\frac{1}{N} \sum_{n=1}^{N} \frac{n}{\mu_{0}}=\frac{N+1}{2 \mu_{0}}
$$

If the capacity of the memory can store $N_{m e m}$ pages and assuming that the most recent transmitted pages are stored, the new maximum access time is :

$$
T_{\max }=\frac{N-N_{m e m}}{\mu_{0}}
$$

and the probability of a randomly selected page not being in memory is

$$
P=\frac{N-N_{m e m}}{N}
$$

Therefore, the new overall average access time is

$$
T_{\text {average_mem }}=\frac{\left(N-N_{m e m}+1\right)\left(N-N_{m e m}\right)}{2 N \mu_{0}}
$$

Let $N_{m e m}=x \cdot N$ where $0 \leq x \leq 1$

Percentage of access time with respect to the original access time is

$$
\begin{aligned}
& T_{\text {percent }}=\frac{T_{\text {average_mem }}}{T_{\text {average }}} \cdot 100 \% \\
& T_{\text {percent }} \approx(1-x)^{2} \quad \text { if } N \gg 1
\end{aligned}
$$


Fig. 2 shows the relationship of the percentage access time versus the memory capacity. The access time decreases quadratically with the memory capacity.

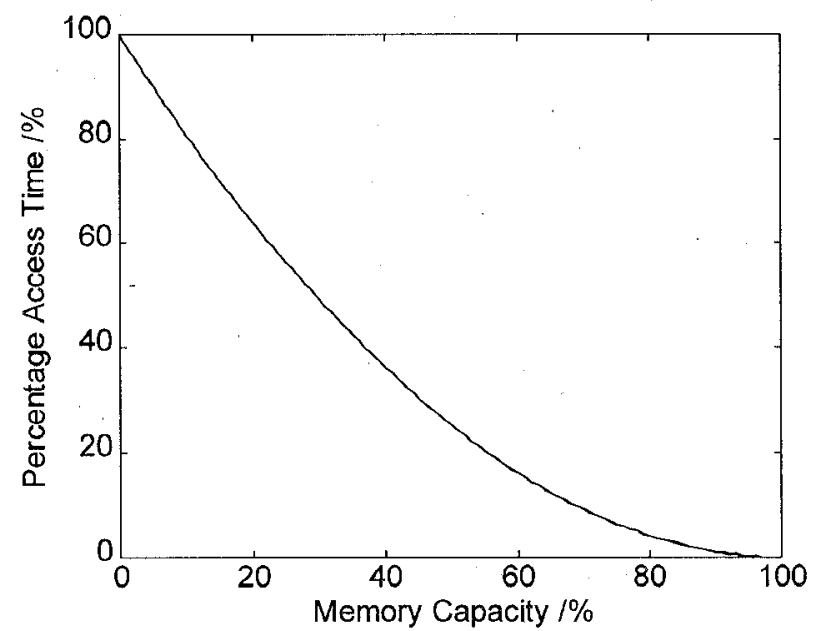

Fig. 2 Percentage Access Time Versus Memory Capacity

\section{B. Reduction of Update Delay}

It would be pointless to have instant access time if the information viewed was not up to date. Therefore, the reductions of update delay is also very important in a real time information system.

The average update delay in all the schemes mentioned in section II is entirely dependent on the broadcasting schedules. This is because any data changes in a page received at the information center can only be delivered to the end users at the next transmission of that page. The update delay does not need to include the transmission time and the retrieval time from local memory because these are negligible as compared with the waiting time for the next transmission.

In our system, the updated information may be broadcast using the ghost rows. The system may be modeled as having two queues as shown in Fig. 3, the main queue is the ordinary level 1 transmission [1][2] which transmits the database continuously and cyclically. The ghost row queue is used to transmit the updated information.

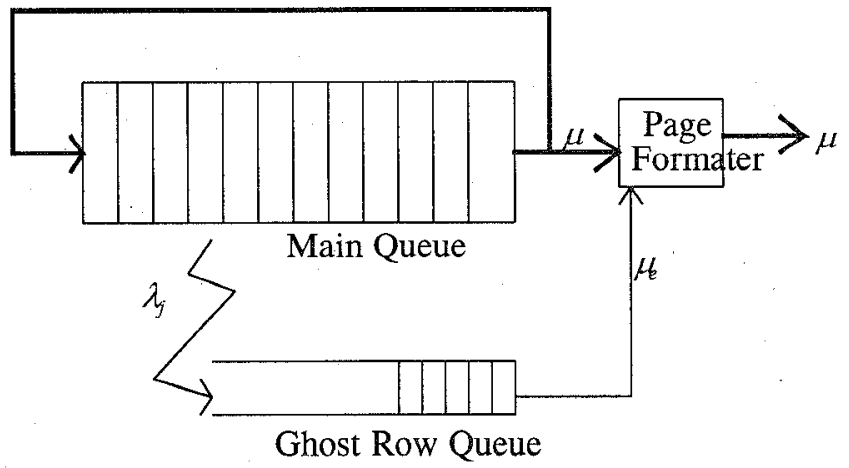

Fig. 3 System Model
If the original transmission rate is $\mu_{o}$ pages per second, the original number of packets per page is $r$ and the number of ghost rows used per page is $e$. The new transmission rate for the main queue and the ghost row queue are $\mu$ pages per second and $\mu_{e}$ packets per second respectively.

$$
\mu=\mu_{0} \cdot \frac{r}{r+e}, \quad \mu_{e}=\mu \cdot e
$$

For users using terminals without the capability of receiving ghost rows and/or without the suitable decoding software, the operation remains unchanged except that the average access time will appear to be slightly longer. The percentage of decrease in transmission rate is

$$
\text { Percentage decrease in transmission rate }=\frac{100 e}{r+e} \%
$$

Since $r \gg e$, the increase in access time is only small.

The ghost row queue model is as shown in Fig. 4. It is modeled as a single server with different types of messages, each type of message represents a row in a teletext page and in a first come first served (FCFS) discipline. The message arrival processes are assumed to be Poisson with arrival rate $\lambda$ packets per second. This is appropriate because the number of pages in a teletext system is normally large. The service time of all message types is $\mu_{e}$ packets per second as defined above.

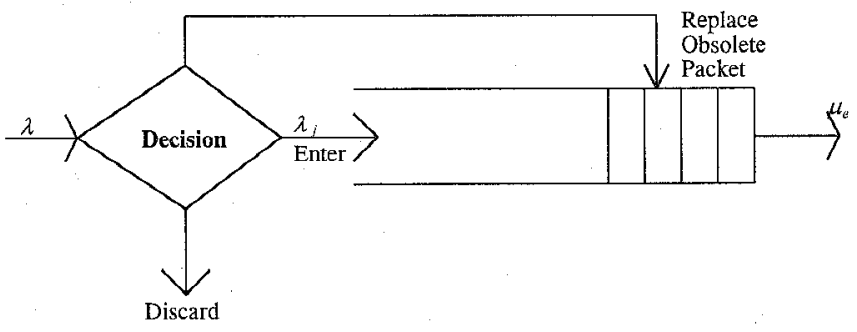

Fig. 4 Ghost Row Queue Model

If the ratio of the arrival rate to the service rate $\rho$ is less than unity $\left(\lambda<\mu_{e}\right)$. Assuming the buffer is of reasonable size and no special technique are being used, the model would be equivalent to an $\mathrm{M} / \mathrm{M} / 1$ model with an infinite buffer [4]. The mean waiting time for $(\rho<1)$ is as follows :

$$
w=\frac{1}{\mu(1-\rho)}
$$

(where $\rho$ is the ratio of the arrival rate to the service rate

$$
\left.\left.\rho=\frac{\lambda}{\mu_{e}}\right)\right)
$$

The queue is reasonably short when $\rho<1$. If $\rho \rightarrow 1$ or even worst $\rho>1$, the queue length and wait time will grow without bounds. This is solved based on the following facts. Since the changed information of a page will eventually reach the users at the next transmission of that page, it would be of no use if the changed information using the ghost row queue arrives later than the next transmission in the main queue. Therefore, if the wait time in the 
ghost row is long and that the time to transmit that packet is later than the next transmission of the page containing this packet, this packet should not be entered into the ghost row queue and should be discarded. Also, any old information in the teletext is always replaced and overwritten by new information. It is therefore, pointless to send a packet waiting in the queue if this packet has already been changed or updated. When a packet arrives and finds that the same packet is already in the queue, this obsolete packet in the queue will then be replaced by the new packet. Because of these two factors, a packet of a page will enter the queue only if the same packet is not already in the queue and the packet will reach the user faster than the next transmission of that page. If the total number of teletext pages is $N$ and is finite, the number of packets in the ghost row queue must be finite. The system therefore reaches a steady state for all values of $\rho$.

Consider a system with a total of $N$ teletext pages and originally $r$ packets in each page. Therefore, the total number of different packets is $N \cdot r$.

If a packet in a page $i$ changes, assume that the time between the occurrence of this change and the next transmission of that page is $\tau$. This packet arrives at the ghost row queue and finds that there are $n$ packets in the queue. The wait time would be $w_{i}=\frac{n+1}{\mu_{e}}$ if this packet enters the system at the end of the queue. Using the above argument, we get the following probability.

$P(n)$ - probability of $n$ messages in system at equilibrium,

$$
P(\text { notq })=\frac{\left(\begin{array}{l}
N \cdot r-1 \\
n
\end{array}\right)}{\left(\begin{array}{l}
N \\
n
\end{array}\right)}=\frac{N \cdot r-n}{N \cdot r} \quad \text { (packet not already in the queue) }
$$

$$
P\left(\tau<=w_{i}\right)=\frac{w_{i}}{T_{N e w c y c}}=\frac{n+1}{N \cdot e}
$$

where $T_{\text {Newcyc }}$ is the new cycle time and $w_{i}$ is the wait time for packet $i$

$$
\begin{aligned}
& T_{\text {Newcyc }}=\frac{N}{\mu} \text { and } w_{i}=\frac{n+1}{\mu_{e}} \\
& P\left(\tau>w_{i}\right)=1-P(\tau<=q)
\end{aligned}
$$

Three cases need to be considered.

Case 1 : that packet is not already in the queue and the wait time $w_{i}$ is shorter than the time required in the main queue $\left(w_{i}<\tau\right)$. This packet enters the system at the end of the ghost row queue.

$$
P(\text { enter })=P(n) \cdot \frac{N \cdot r-n}{N \cdot r} \cdot\left(1-\frac{n+1}{\mu_{e}}\right)
$$

Notice that $P\left(\tau<=w_{i}\right)=1$ when $n=N \cdot e-1$,i.e. the updated packet will never enter the ghost row queue, therefore, the maximum number of packets in the ghost row queue is $N \cdot e-2$.

Case 2 : that packet is not already in the queue and the wait time $w_{i}$ is longer than or equal to the wait time required for the next transmission in the main queue $\left(w_{i}>=\tau\right)$. This packet is discarded and the updates depend on the normal transmission in the main queue.

$$
P(\text { discard })=P(n) \cdot \frac{N \cdot r-n}{N \cdot r} \cdot\left(\frac{n+1}{N \cdot e}\right)
$$

Case 3 : that packet is already in the queue in position $\mathrm{k}$, new packet replaces the old packet and remains in the kth position. $k=1$ to $N \cdot e-2$.

Note that the probability of $\left(w_{i}<\tau\right)=1$, otherwise this packet would not have entered the queue in the first place.

$$
P(\text { replace })=P(n) \cdot\left(\frac{n}{N \cdot r}\right)
$$

To calculate $P(n)$, consider the system with $n$ packets in an equilibrium state. (Note: this system is always in equilibrium as discussed above). From [9], we have

$$
P(n)=P(0) \frac{\lambda_{0} \lambda_{1} \lambda_{2} \ldots \lambda_{n-1}}{\mu_{1} \mu_{2} \mu_{3} \ldots \mu_{n}}=P(0) \prod_{j=0}^{n-1} \frac{\lambda_{j}}{\mu_{j+1}}
$$

There are no two packets of the same type in the queue simultaneously and the number of packets in the queue must not exceed $N \cdot e-2$. Therefore,

$$
\begin{aligned}
& \lambda_{j}=\lambda \cdot P(\text { no type j packet }) \cdot P(w<\tau) \\
& \begin{cases}\lambda_{j}=\lambda \cdot\left(\frac{N \cdot r-j}{N \cdot r}\right) \cdot\left(1-\frac{j+1}{N \cdot e}\right) & j<=N \cdot e-2 \\
\lambda_{j}=0 & j>N \cdot e-2\end{cases} \\
& \mu_{j}=\mu=\mu_{0} \cdot e ; \quad j=1,2, \ldots(N \cdot e-2)
\end{aligned}
$$

Hence, we get that

$$
\begin{aligned}
& \left\{\begin{array}{l}
P(n)=P(0) \cdot\left(\frac{\rho}{N \cdot r}\right)^{n} \cdot\left(1-\frac{n+1}{N \cdot e}\right)^{n} \cdot \frac{(N \cdot r) !}{(N \cdot r-n) !} \\
P(n)=0
\end{array}\right. \\
& n<=N \cdot e-2 \\
& n>N \cdot e-2 \\
& \text { Since } \quad \sum_{n=0}^{N \cdot e-2} P(n)=1 \\
& \text { We get } P(0)=\left[\sum_{n=0}^{N \cdot e-2}\left(\frac{\rho}{N \cdot r}\right)^{n} \cdot\left(1-\frac{n+1}{N \cdot e}\right)^{n} \cdot \frac{(N \cdot r) !}{(N \cdot r-n) !}\right]^{-1}
\end{aligned}
$$

The waiting time for the packet will be $(n+1) / \mu_{e}$ if the packet is allowed to enter the queue. If the packet is discarded, the refresh delay would rely on the next transmission of the page containing this packet which is equal to $\tau$. Otherwise the delay would be $k / \mu_{e}$ if the packet is already in the queue at position $k$.

Therefore, the mean waiting time for this packet is 


$$
w_{i}=\sum_{n=0}^{N \cdot e-2}\left[\begin{array}{l}
\frac{n+1}{\mu_{e}} \cdot P(\text { enter })+\frac{1}{N} \sum_{t=\frac{1}{u}}^{\frac{N}{u}} \tau \cdot P(\text { discard })+ \\
\sum_{k=1}^{n} \frac{k}{\mu_{e}} \cdot \frac{1}{n} \cdot P(\text { replace })
\end{array}\right]
$$

Assume that all packets have the same probability of changing. This is appropriate if a long time frame is considered. The mean update delay over all message types is

$$
U_{\text {delay }}=\sum_{i=1}^{N} P_{i} \times w_{i}=w_{i}
$$

Substitute (14),(15), (16) and (17) into (23), we get

$$
U_{\text {delay }}=\sum_{n=0}^{N \cdot e-2} P(n) \cdot\left\{\frac{n+1}{2 \cdot \mu \cdot e \cdot N \cdot r}\left[\begin{array}{l}
2(N \cdot r-n)\left(1-\frac{n+1}{N \cdot e}\right)+ \\
\frac{(N+1)(N \cdot r-n)}{N}+n
\end{array}\right]\right\}
$$

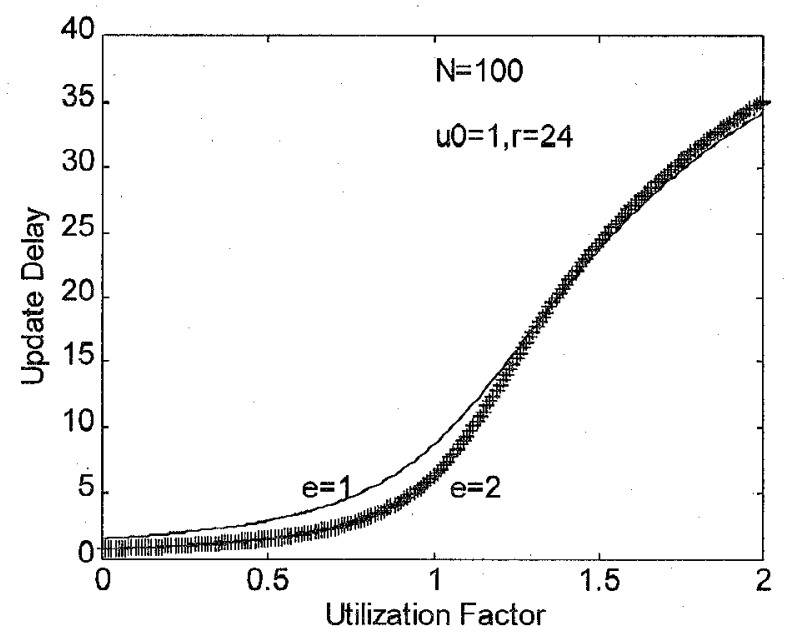

Fig. 5 Update Delay $U_{\text {delay }}$ Versus Utilization Factor $\rho$

A quantity of interest in this system is the probability of a packet being discarded. This happens when a packet arrives at the ghost row queue and finds that it is not already in the queue and the wait time is longer than or equal to the time required in the main queue. The update of this packet will rely on the next transmission of the page containing this packet and therefore would not get any benefit from this system. From (16), the probability of discard for any message is given by

$$
P(\text { discard })=\sum_{n=0}^{N \cdot e-2} P(n) \cdot \frac{N \cdot r-n}{N \cdot r} \cdot\left(\frac{n+1}{N \cdot e}\right)
$$

The discard probability is shown as a function of the utilisation actor $\rho$, for several values of $e$ in Fig. 6 . As expected, the discarding probability increases with increases in load and decreases as the number of ghost rows increases.
The worst case is when the arrival rate far exceeds the transmission rate (i.e., $\lambda \rightarrow \infty$ ). The maximum queue length is $N \cdot e-2$ and the discard probability is as follows :

$$
\operatorname{Lim}_{\lambda \rightarrow \infty} P(\text { discard })=\frac{N(r-e)+2}{N \cdot r} \cdot \frac{N \cdot e-1}{N \cdot e}
$$

This probability is approaching one because $(r>>e \& N>>1)$ but never equal to one. Therefore, even in the absolute worst case, some benefits are still maintained. Also, this worst case does not happen in teletext as the system is designed for human reception.

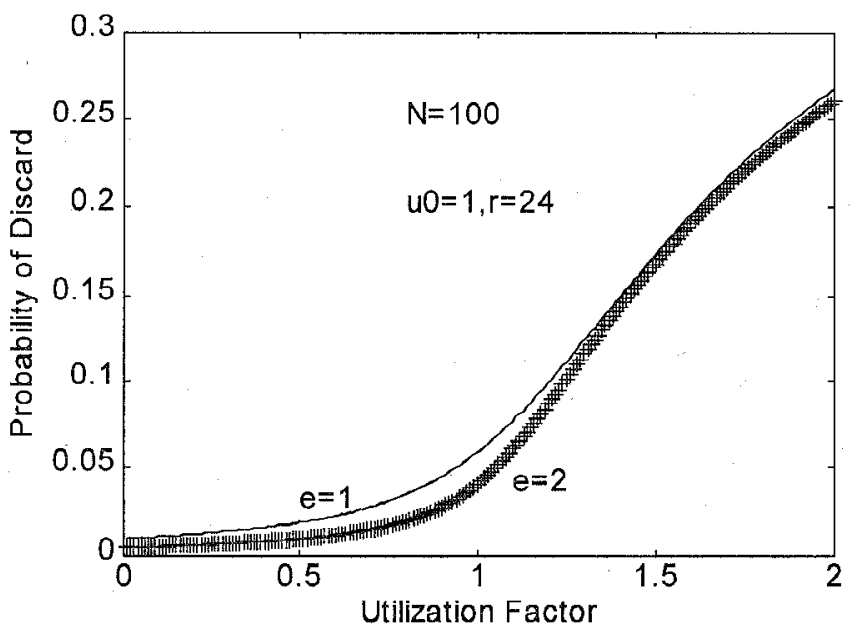

Fig. 6 Probability of discard $P($ discard $)$ Versus

Utilization Factor $\rho$

\section{Numerical examples and discussions}

Consider a teletext system with a 100 pages and the transmission rate $\mu_{0}$ is 1 . ( $\mu_{0}$ varies from 1 if one TV line is used in VBI transmission to around 650 if all lines are used in full channel transmission. [2]). The original number of packets per page $r=24$ as in normal teletext transmission [2]. Using (2), the average access time and update delay without using any background memory is 50.5. Fig. 2 shows the access time versus memory capacity. Fig, 5 shows the update delay versus $\rho$ with different number of $e$. It shows that significant improvement on update delay can be achieved using this method. As expected, the improvement reduces with increasing $\rho$, because as $\rho$ increases, the probability of discard increases as shown in Fig. 6.

From Fig. 7, the percentage improvement in update delay is a function of $\rho$. If $\rho$ is large, this system would not be very effective. As discussed above, $\rho$ is normally small because teletext is designed for human reception and therefore the data change rate would not be too fast. Take the teletext system being used by the Stock Exchange of Hong Kong as an example. They use full channel transmission in PAL format, the transmission rate $\mu_{0}$ is around 600 pages per second. This system was monitored during an active trading day with turnover over 100 billion Hong Kong dollars and it was found that the average data change rate was below $10 \mathrm{kbit} / \mathrm{s}$. If the number of ghost row used per page in this new system is $e=1$, the transmission rate for the ghost row queue $\mu_{e}=600$ packets per second or $192 \mathrm{kbit} / \mathrm{s}$. This is equivalent to $\rho<0.1$ which is very small and therefore significant 
improvement can be achieved. It is anticipated that the data change rate for most other real time teletext system would also be around this region.

For users using older terminals without the capability of decoding the ghost rows, using (9), the average access time and update delay with $e=1$ is only increased by around $4 \%$ which is hardly noticeable. We can conclude that the new system is also downward compatible to the older equipment.

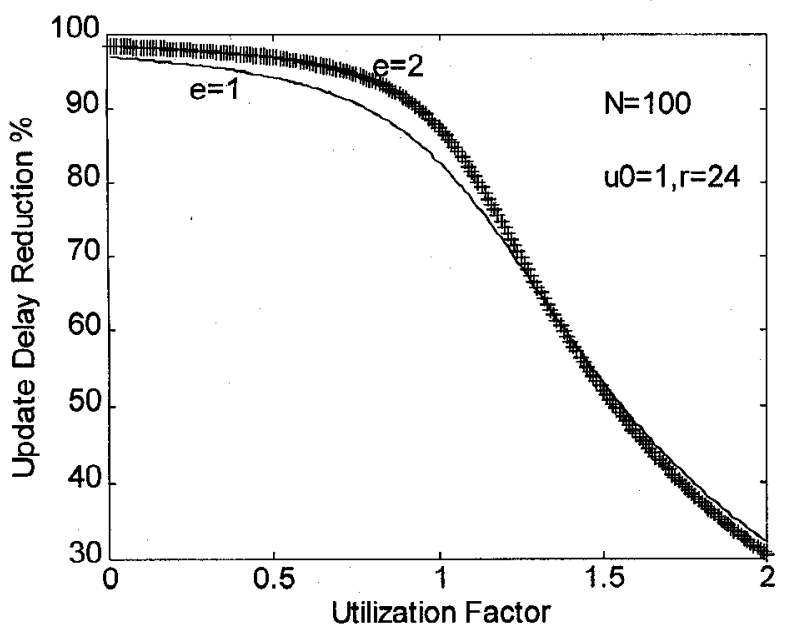

Fig. 7 Percentage Reduction in Update Delay Versus Utilization Factor $\rho$

\section{Conclusions}

Both the access time and the update delay are important factors contributing to the quality of services of the broadcast systems. We have analyzed the performance of using ghost rows and modern receivers with storage capabilities to shorten these delays using full channel teletext as an example. The results showed that significant improvement can be achieved. In addition, older equipment may still be used in the new system with very little degradation. The same algorithm may apply to other wide band systems with the ghost rows replaced by a small secondary channel.

\section{References}

[1] Gecsei, "The Architecture of Videotex Systems," Englewood Cliffs, NJ: Prentice-Hall, 1983.

[2] Pim, D. N., "Television and Teletext", Macmillan Education Ltd, 1988.

[3] Kinghormn J. R., "New Features in World System Teletext," IEEE Transaction on consumer Electronics, Vol. CE-30, Aug., 1984, pp. $437-441$.

[4] Kinghorn J.R., Philips Components Report No. MTV89008 "Enhanced Computer Controlled Teletext SAA5243 Series User Manual".
[5] Ammar, M. H. and Wong, J.W., 'The Design of Teletext Broadcast Cycles", Performance Evaluation, Vol.5, Nov. 1985, pp. 235-242.

[6] Ammar, M. H. and Wong, J.W., On The Optimality of Cyclic Transmission in Teletext Systems", IEEE Transactions on Communications, Vol. COM-35, No. 1, January 1987. pp 6873.

[7] Sriskanthan N. and Subramanian K.R., Design of a PC-Based Teletext Terminal, IEEE Transaction on Consumer Electronics, Vol. 37, No. 4, November 1991, pp. 918-925.

[8] Siu,Y.M., Chan C.K. and Ho K.L., Rebroadcasting of Real Time Teletext Data, IEEE Transaction on Consumer Electronics, Vol. 39, No. 2, May 1993, pp. 131-135.

[9] Kleinrock, Leonard, Queueing Systems, Vol. 1: Theory, John Wiley \& Sons, 1975.

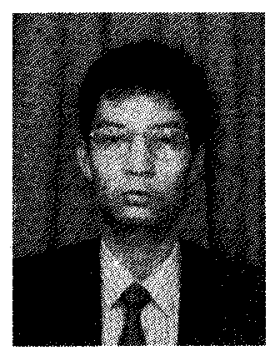

Yun Ming Siu received the B.Sc. degree in 1981 from the University of Manchester, UK and the Ph.D. degree from the University of Hong Kong in 1996. From 1981-1989, he worked at Racal-BCC, UK, first as a development Engineer, and then as a team leader responsible for the development of frequency hopping radio and Tactical communications systems. In 1990, he joined the Department of Electronic Engineering at the City University of Hong Kong as a Lecturer. His current research interests include information broadcasting and delivery systems, and communication networks. He is a Chartered Engineer of the Engineering Council, UK, a member of the IEE, IEEE, Chinese Institution of Electronics and the Hong Kong Institution of Engineers.

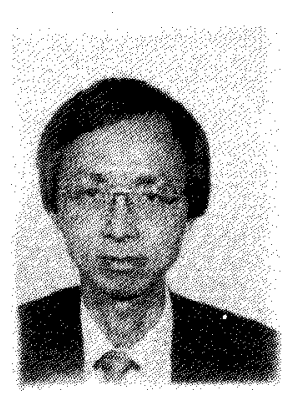

Chok Ki CHAN received the B.S. and M.S. degrees both in E.E. from University of California, Los Angeles in 1977 and 1978 respectively. He received the Ph.D. degree in Electronics from the Chinese University of Hong Kong in 1984. In 1978 and 1979, he was with the Severe Environment Systems Co. Ltd., California, as a design engineer of computer systems. From 1980 to 1985, he was with the faculty of the

Department of Electronic Engineering, Hong Kong Polytechnic. He is currently a Reader in theDepartment of Electronic Engineering, City University of Hong Kong. His current research interests include image compression, signal processing and information delivery. Dr. Chan is a Chartered Engineer of the Engineering Council, U.K. and a member of the IEE, IEEE and the Hong Kong Institution of Engineers, Currently, he is the Chairman of the IEEE Hong Kong Section. 


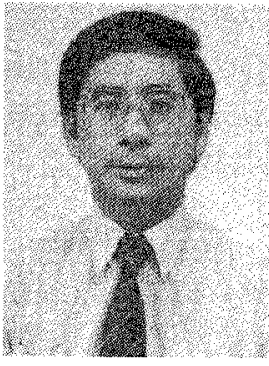

Ka Leung Ho received the B.Sc.(Eng.) degree and the M.Phil. degree in Electrical Engineering from the University of Hong Kong in 1971 and 1973, respectively, and the Ph.D. degree from the University of London in 1977. In 1984, he joined the Department of Electrical and Electronic Engineering at the University of Hong Kong and is currently a senior lecturer. His current research interests include millimeter wave propagation, signal processing and communication systems. Dr. Ho is a member of IEEE. 